\title{
Oral immunotherapy for the treatment of peanut allergy: systematic review of six case series studies
}

\author{
*Aziz Sheikha, Ulugbek Nurmatova, Iris Venderboscha, Erik Bischoff ${ }^{b}$ \\ a Allergy and Respiratory Research Group, Centre for Population Health Sciences, The University of Edinburgh, Edinburgh, UK \\ ${ }^{b}$ Nijmegen Medical Centre, Primary and Community Care, Radboud University, Nijmegen, The Netherlands
}

Originally received 22nd November 2010; resubmitted 4th M arch 2011; revised 3rd April 2011; accepted 4th April 2011; online 21st September 2011

\begin{abstract}
Background: Allergy to peanuts is associated with considerable morbidity and, in a minority of cases, mortality. Natural resolution to peanut allergy occurs in only a few cases, hence the need to find effective interventions. Peanut oral immunotherapy (OIT) is a potentially important new therapeutic development.

Aims: To assess the benefits and harms of OIT for peanut allergy.

Methods: Fourteen databases were searched for published reports and unpublished/in-progress studies. We included studies employing randomised controlled trial (RCT), quasi-RCT, controlled clinical trial, controlled before-and-after, interrupted time series, and case series designs.

Results: Six studies enrolling a total of 85 participants satisfied our inclusion criteria. All studies employed a case series design and were thus judged to be at high risk of bias. Overall, this body of evidence provided suggestive evidence that it is possible for many participants to increase their threshold dose for peanut exposure whilst receiving treatment. Adverse reactions were common and, whilst most of these were relatively minor, some were potentially life-threatening.

Conclusions: OIT appears to be a potentially promising new therapy for the short- to medium-term management of carefully selected and monitored patients with peanut allergy. The effectiveness and cost-effectiveness of OIT - particularly over the longer term - need to be clearly established using more robust designs before its clinical use can be contemplated. Given the risk of triggering serious adverse reactions, OIT should not be administered outside clinical trial settings.

(C) 2012 Primary Care Respiratory Society UK. All rights reserved.

A Sheikh, et al. Prim Care Respir J 2012; 21(1): 41-49

http://dx.doi.org/10.4104/pcrj.2011.00071
\end{abstract}

Keywords oral immunotherapy, peanut allergy, systematic review, treatment

See linked editorial by Lötvall \& Calderón on pg 7

The full version of this paper, with online Appendices 1-7, is available online at www.thepcrj.org

\section{Introduction}

Food allergies affect $4-8 \%$ of children and $1-2 \%$ of adults. These reactions occur most often in young children and in individuals with a personal or family history of atopic disorders. The majority of children outgrow the most common food allergies (i.e. to cows' milk and hens' eggs) and these can then safely be (re)introduced when they are older; in contrast, peanut allergy tends to be lifelong.

Studies have shown that in western countries peanut allergy is no longer an uncommon disease, with recent estimates from English general practices revealing that over 25,000 people now have a GP-recorded diagnosis of peanut allergy. ${ }^{2}$ Concern about the prevalence of peanut allergy in such countries is rising, ${ }^{2}$ as is people's awareness of the dangers associated with peanut allergy. It is in this respect important to appreciate that, whilst all food allergies have the potential to induce anaphylaxis, some foods are more likely than others to cause potentially lifethreatening reactions. ${ }^{3}$ Among anaphylactic reactions attributed to foods, $23-47 \%$ are triggered by peanut protein. ${ }^{4}$

It has been suggested that ingestion of peanuts by mothers during pregnancy or while lactating could be a potential risk factor for the development of peanut allergy, but the evidence for this is weak. ${ }^{5,6}$ More plausibly, following the observation that

\footnotetext{
* Corresponding author: Professor Aziz Sheikh, Centre for Population Health Sciences, University of Edinburgh, Doorway 3, M edical School, Teviot Place, Edinburgh, EH8 9DX, UK. Tel: +44 (0)131 6514151 E-mail: aziz.sheikh@ed.ac.uk
} 
the risk of peanut allergy is increased in children with atopic dermatitis who have had peanut oil-containing creams applied to inflamed skin, ${ }^{7}$ it has been suggested that cutaneous exposure may be particularly important in inducing sensitisation and its subsequent clinical expression, whereas oral and/or mucosal exposure to peanuts may encourage the development of immunological tolerance..$^{8}$ This remains an active line of enquiry, particularly in relation to those with filaggrin gene mutations. ${ }^{9}$

Most peanut allergic reactions have the characteristic hallmarks of typical IgE-mediated reactions in that clinical symptoms develop within seconds or minutes of exposure and can be triggered by very small quantities of peanut protein. ${ }^{4,10,11}$ The clinical expression of peanut allergy may include features of urticaria, angio-oedema, vomiting, diarrhoea, wheezing, throat tightness, dyspnoea, and cardiovascular collapse. ${ }^{12}$

Meticulous avoidance of peanuts and peanut-containing products in sensitised individuals is currently the cornerstone of management. This is, however, far from straightforward given the now widespread use of peanuts in a variety of foods. ${ }^{13-15}$ The quality of life of affected individuals may be reduced because of constant fear over food choices and the associated risk of triggering severe reactions including anaphylaxis. ${ }^{16}$ People with peanut allergy therefore also need to be trained in emergency selfmanagement with adrenaline (epinephrine) auto-injectors and/or antihistamines if accidental exposure occurs. ${ }^{17,18}$ There is currently no cure for peanut allergy, so there is therefore a pressing need to develop safe and effective curative interventions.

As early as 1908 Schofield showed that immunotherapy can lead to desensitisation in patients with food allergy. ${ }^{19}$ Immunotherapy - which refers to the deliberate and controlled exposure of the allergic individual to very small but progressively increasing doses of the allergen in question until a maintenance dose is reached - has in the intervening period become an established therapeutic approach for patients with, for example, pollen and venom allergy, but this is not yet the case for those with food allergy. This is because the most commonly used route of delivery (i.e. subcutaneous) is effective and - if carefully administered - safe in treating some forms of inhalant and stinging insect allergen sensitivity ${ }^{20,21}$ but, because of the high risk of adverse events, it is not yet deemed safe for use in the management of food allergy. ${ }^{22}$

Since then, alternative methods of delivering immunotherapy have been developed, including the use of oral immunotherapy (OIT). OIT is of particular interest as it has the potential to improve the community-based management of children with food allergy, many of whom also have relevant food allergyrelated co-morbidities such as asthma. ${ }^{23}$ It involves initially giving very small doses of peanut protein via the oral route and gradually increasing this until a more substantial dose is achieved, which is then maintained over time. The aim of this treatment approach is first to induce a state of desensitisation to the peanut allergen - which can thus confer a degree of protection against the dangers of accidental exposure whilst on treatment - and then to induce longer-term tolerance. ${ }^{2}$ Doubleblind placebo-controlled food challenges (DBPCFCs) and/or open food challenges can be used to assess whether desensitisation and tolerance have been achieved.

OIT is therefore a potentially important new treatment approach for people with peanut allergy which, if successful, could help to minimise the risks associated with accidental exposure to peanuts and peanut-containing foods. However, at present there is still considerable uncertainty about the effectiveness and safety of this approach. We therefore sought systematically to identify, critically appraise, and summarise the available evidence on the benefits and harms associated with OIT for the management of people with peanut allergy.

\section{Methods \\ Systematic review protocol}

The methods used in this review were specified in advance and documented in a protocol which is available on request from the corresponding author.

\section{Criteria for considering studies for this review}

\section{Types of studies}

The following study types were eligible for inclusion: randomised controlled trials (RCTs); quasi-RCTs; controlled clinical trials (CCT); controlled before-and-after designs; interrupted time series; and case series. Our working definitions for different study designs are detailed in Appendix 1 in the Supplementary materials (available at www.thepcrj.org). ${ }^{24,25}$

\section{Types of participants}

We were interested in studies on patients with confirmed peanut allergy of all ages, both sexes, and any ethnic group. We used the following working definition of peanut allergy: a history of significant clinical symptoms within 60 mins after the ingestion of peanuts and objectively confirmed peanut sensitivity as ascertained by a positive skin prick test response to peanut allergen (which was defined as a wheal of $\geq 3 \mathrm{~mm}$ larger than that produced by the saline control) $)^{26}$ or the presence of specific IgE to peanut (which was defined as a positive in vitro serum peanut IgE result of $>7 \mathrm{kU} / \mathrm{L}$ for children $\leq 2$ years of age and $>15$ $\mathrm{kU} / \mathrm{L}$ for children $>2$ years of age). ${ }^{27}$

\section{Types of interventions}

Interventions of interest were OIT in patients with confirmed peanut allergy compared with a placebo group, with an alternative way of administering desensitisation, or without a comparison group. We were interested in standard approaches to delivering immunotherapy (i.e. incorporating escalation, buildup, and maintenance phases) and any variants of this approach (e.g. rush immunotherapy).

\section{Types of outcome measures}

a) Primary

Our main outcomes of interest were evidence of desensitisation and tolerance. Desensitisation was defined as an increase in the amount of peanut allergen to which peanut-allergic patients could be exposed before the induction of symptoms, whilst still 
receiving OIT. Tolerance was defined as the ability to consume peanuts safely after OIT had been discontinued and evidence of accompanying long-term immunological changes.

b) Secondary

Secondary outcome measures of interest included:

- Satisfaction (patients, parents)

- Changes in quality of life

- Health care utilisation

- Cost-effectiveness

- Attrition rate

- Adverse events (together with an assessment of their severity using the classification scheme detailed below and associated medication use)

- Immunological changes

We assessed the severity of systematic reactions with the grading system proposed in the Position Paper of the European Academy of Allergology and Clinical Immunology on Immunotherapy: ${ }^{28}$

- Grade 1: Non-specific reactions: reactions probably not lgEmediated (i.e. discomfort, headache, arthralgia, etc).

- Grade 2: Mild systemic reactions: mild rhinitis and/or asthma (peak expiratory flow rates (PEFR) $>60 \%$ of predicted or of the personal best values) responding adequately to antihistamines or inhaled $\beta_{2}$-agonists.

- Grade 3: Non life-threatening systemic reactions: urticaria, angio-oedema, or severe asthma (PEFR $<60 \%$ of predicted or of personal best values) responding well to treatment.

- Grade 4: Anaphylactic shock: rapidly evoked reaction of itching, flushing, erythema, bronchial obstruction, etc requiring intensive treatment.

\section{Search methods for identifying studies}

We attempted to identify all relevant studies regardless of language, geographical area or publication status (published, unpublished, in press, and in progress). We focused exclusively on human studies.

\section{Electronic databases}

EMBASE, PubMed, Cochrane Library, ISI Web of Science, Biosis, Global Health, AMED, TRIP, CAB, CINAHL, LILACS, PakMediNet, IndMed, and Google Scholar were searched for studies reported in the period 1990-2010 using the search terms detailed in Appendices 2-5 (see Supplementary materials). Our last searches were run on 16 February 2010.

\section{Other searches}

Unpublished work and ongoing research was found by searching key Internet-based relevant databases: Current Controlled Trials (http://www.controlled-trials.com) and ClinicalTrials.gov (http://www.clinicaltrials.gov) and the abstract books of recent scientific conferences. We contacted three experts in the field for details of ongoing and unpublished work (see Appendix 6 in the Supplementary materials).

Reference lists

The reference lists of all studies identified by the above methods were checked for additional studies of possible relevance.

\section{Selection of studies}

Two reviewers (IV and UN) independently reviewed the titles and/or abstracts and selected all studies that potentially satisfied our inclusion criteria. These two authors then independently assessed the full text copies of the potentially eligible studies against the inclusion criteria. Where the authors agreed, they either included or excluded the trial. Any disagreements were resolved by discussion between the reviewers but, if they were unable to come to agreement, a third reviewer (AS) arbitrated.

\section{Data collection, quality assessment, and synthesis of} data

Two reviewers (IV and UN) independently extracted data using a customised data extraction form. We used the quality assessment form produced by the National Institute for Health and Clinical Excellence (NICE) to help critically appraise case studies. ${ }^{24}$ Any disagreement between the reviewers was resolved by discussion. If no agreement could be reached, a third reviewer (AS) arbitrated.

Information was extracted from each included study on the following study parameters:

- Inclusion and exclusion criteria

- Characteristics of study participants (including age, gender, IgE level, co-morbidities)

- Type of intervention (including dose, frequency, and duration of OIT)

- Outcome measures of interest

The data from the included case series were descriptively analysed and a narrative synthesis of findings was undertaken.

\section{Results}

Our searches of the major biomedical databases identified a total of 1,672 potentially eligible studies. After removing duplicates, 1,059 remained. Of these, 1,010 studies were discarded because scrutiny of the titles clearly revealed that they failed to meet the inclusion criteria. Forty-nine potentially appropriate abstracts were reviewed, of which 38 were excluded for not meeting the inclusion criteria. The full text of the remaining 11 studies was examined in more detail. In addition, nine reports were added as potentially relevant after searching the conference proceedings of recent scientific meetings. After carefully reviewing these 20 papers/abstracts, 14 reports were excluded for not meeting the inclusion criteria. Three full-text articles and three abstracts relating to a total of six studies satisfied our inclusion criteria and were therefore included in this review (see the PRISMA flow diagram in Figure 1 and Table 1). ${ }^{29-34}$ We also found several ongoing studies that had not yet reported on any of the outcomes of interest (Table 2).

\section{Description of studies, participants, and interventions \\ Study designs}

All six studies finally selected for the review were case series. ${ }^{29-34}$ The duration of maintenance treatment and the follow-up period varied considerably between studies, ranging from 6 
Figure 1. PRISMA flow diagram

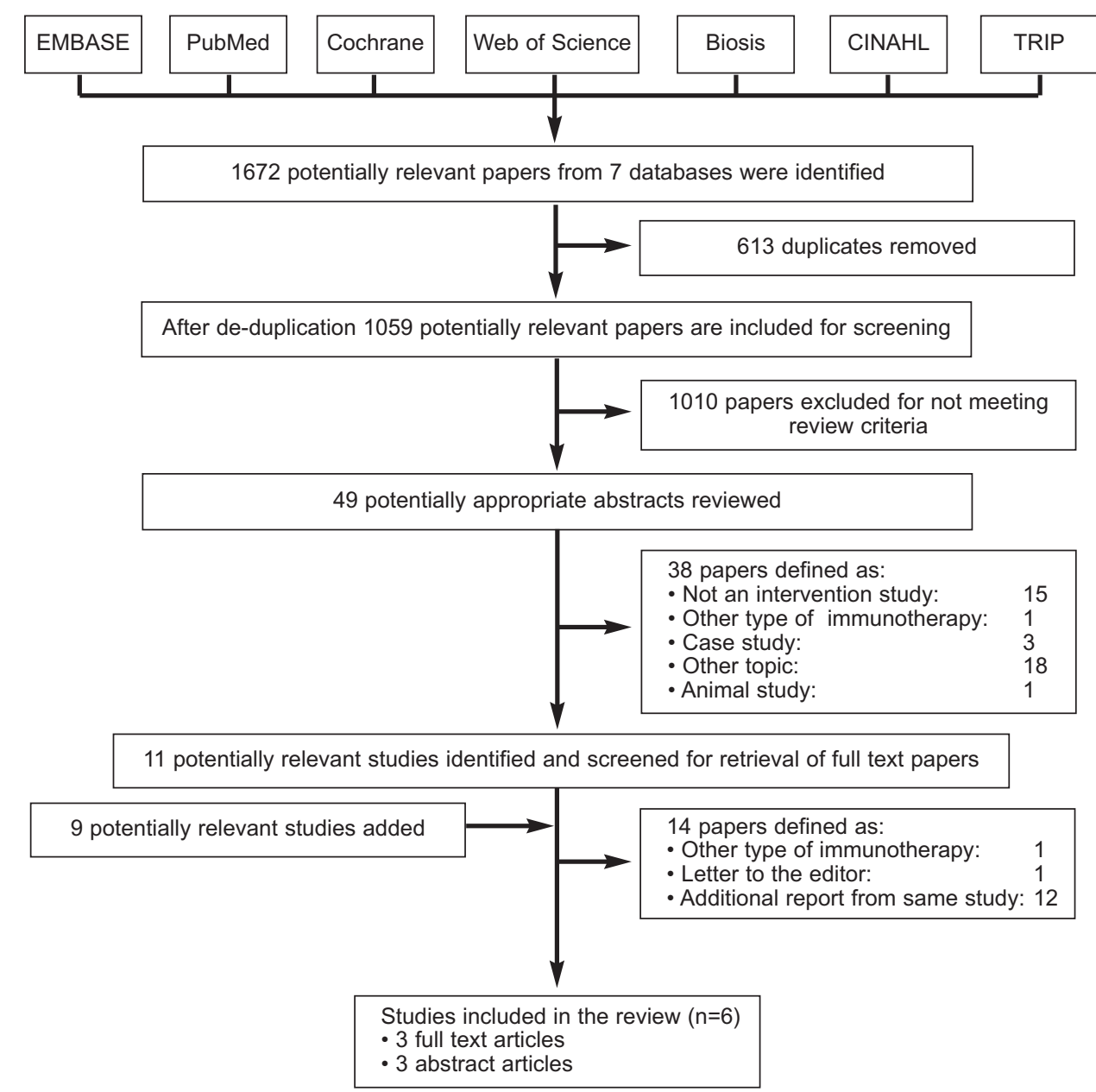

days ${ }^{31}$ to 36 months. ${ }^{29}$ One study described the minimum length of the study period (i.e. 4 days) but did not mention the maximum duration of therapy ${ }^{32}$

Four studies ${ }^{29,32-34}$ were multicentre, one of which was conducted in a private office-based setting..$^{32}$ The two other studies were based in a single centre. ${ }^{30,31}$ Four of the studies were conducted in the USA, ${ }^{29,323-34}$ one in the $\mathrm{UK}^{30}$ and one in Germany. ${ }^{31}$

\section{Participants}

The included studies enrolled a total of at least 85 participants. The main inclusion criteria were a clinical history of reaction to peanut and objectively confirmed peanut allergy as evidenced by an elevated level of peanut-specific lgE and/or a positive skin prick test. The study by Wasserman et al..$^{32}$ differed in that the inclusion criterion was either a clinical history of peanut allergy or objective evidence of peanut sensitisation; also relevant is that this report relates to OIT being administered to patients with a range of food allergies so, in keeping with the focus of this review, we have concentrated solely on the data on patients with peanut allergy. Three studies ${ }^{31,32,34}$ included participants who were judged to have severe peanut allergy and consequently were at high risk of subsequent anaphylactic reactions.

Five of the studies investigated children ${ }^{29-31,33,34}$ and the age of the participants was unknown in one study. ${ }^{32}$

Three studies ${ }^{29-31}$ reported on co-morbidities. All of the participants ( $n=6)$ in the study by Blumchen et al..$^{31}$ had asthma. In the study by Clark et al. ${ }^{30}$ all of the participants $(n=4)$ had a history of eczema. In the study by Jones et al. ${ }^{29}$ most of the participants also had co-morbidities: $92 \%(n=34)$ had an allergic disease other than food allergy (including atopic dermatitis $(69 \%)$, asthma (62\%), and allergic rhinitis (62\%)) and 54\% had an additional food allergy.

In four studies $29,30,33,34$ OIT was given in three phases (escalation, build-up, and maintenance phases). An example of such a three-phase OIT protocol as used by Jones et al..$^{29}$ is given below:

- Initial escalation day: All participants received a first dose of $0.1 \mathrm{mg}$ peanut protein. Every 30 mins the dose was doubled, up to $50 \mathrm{mg}$. The highest tolerated single dose was the starting dose for the build-up phase, which started the following day.

- Build-up phase: Participants had to ingest the daily dose of 
Table 1. Summary of study characteristic.

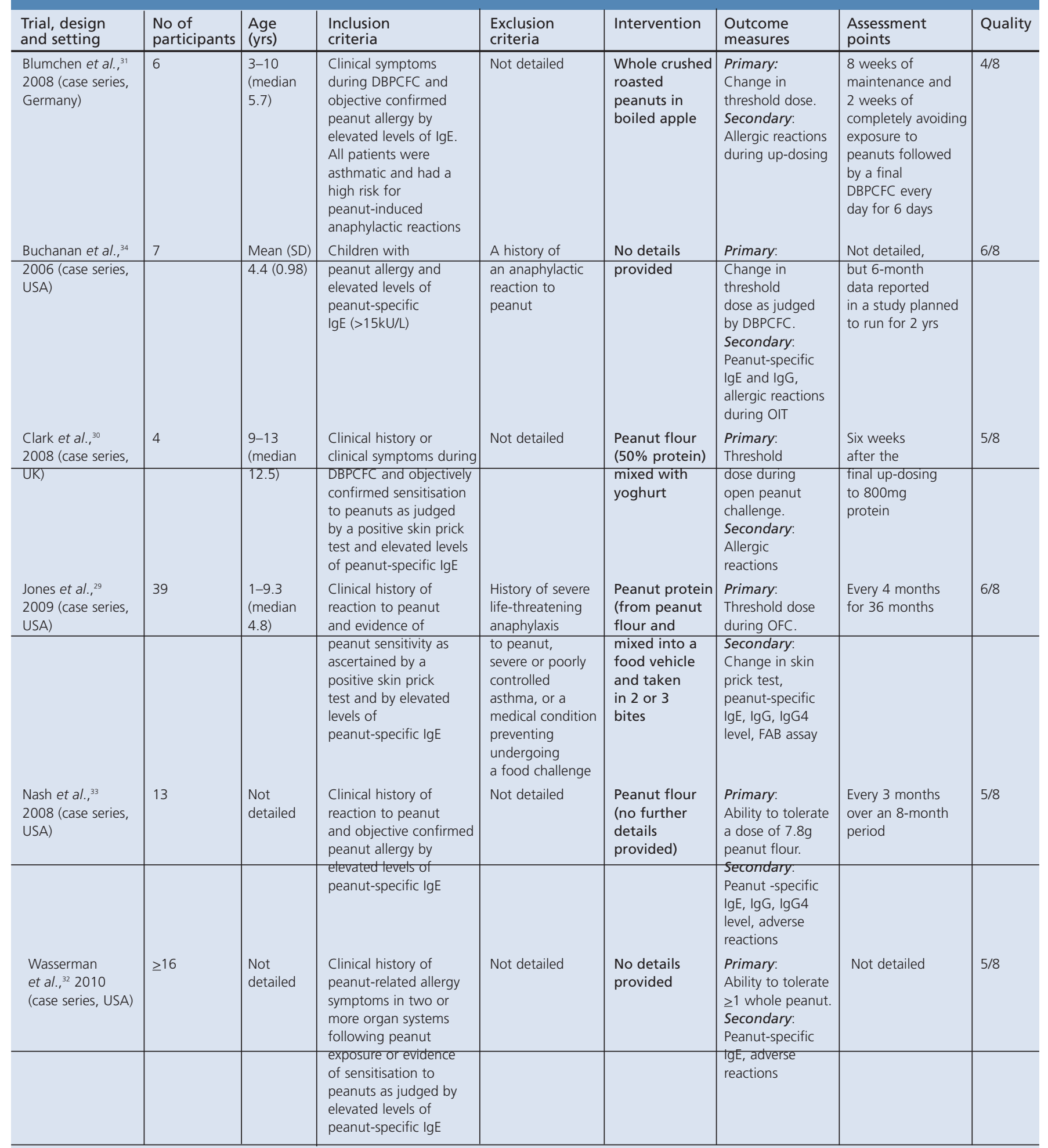

$\mathrm{OFC}=$ oral food challenge, DBPCFC=double-blinded placebo-controlled oral food challenges.

peanut protein in two or three bites a day. Doses were increased by $25 \mathrm{mg}$ every 2 weeks until 300mg was reached.

- Maintenance phase: After reaching 300mg peanut protein daily, participants continued this dose until the oral food challenge (OFC). After this challenge, participants were increased to a daily OIT dose of 1,800mg (if the peanut IgE remained $>2 \mathrm{kU} / \mathrm{L}$ after 12 months on the maintenance dose). 


\begin{tabular}{|c|c|c|c|c|c|c|}
\hline Research team & Setting & Study design & $\begin{array}{l}\text { No. of } \\
\text { participants }\end{array}$ & Intervention & $\begin{array}{l}\text { Started } \\
\text { reporting }\end{array}$ & $\begin{array}{l}\text { Estimated date for } \\
\text { reporting final results }\end{array}$ \\
\hline Burks et al. & Research & Open label & 20 & $\begin{array}{l}\text { Peanut OIT } \\
\text { Open intervention }\end{array}$ & Yes & July 2012 \\
\hline Burks et al. & Research & $\mathrm{RCT}$ & 60 & $\begin{array}{l}\text { Peanut OIT } \\
\text { RCT }\end{array}$ & Yes & November 2013 \\
\hline Clark et al. & Research & Pilot open & 22 & $\begin{array}{l}\text { Peanut OIT } \\
\text { Open intervention }\end{array}$ & Yes & June 2010 \\
\hline Clark et al. & Research & RCT & 104 & $\begin{array}{l}\text { Peanut OIT } \\
\text { RCT } \\
\text { Open intervention }\end{array}$ & No & December 2012 \\
\hline Wood et al. & Research & $\mathrm{RCT}$ & 15 & $\begin{array}{l}\text { Peanut OIT } \\
\text { RCT }\end{array}$ & No & January 2013 \\
\hline
\end{tabular}

In one study ${ }^{32}$ the OIT protocol comprised only two phases: an initial day escalation phase and a build-up phase. Details of the OIT protocol used in this study are given below:

- Initial escalation day: Participants received increasing doses of peanut protein until an allergic reaction occurred; the starting dose used was, however, unclear.

- Build-up phase: Participants ingested the last tolerated dose three times a day for at least 4 days and then received an increasing dose. Doses were increased until the participant was able to tolerate peanuts without restriction (judged as at $\geq 1$ peanut)

One of the studies employed a 'rush protocol' which involved administering increasing doses of peanut dose 3-4 times a day over a period of 6 days. ${ }^{31}$

In three studies ${ }^{29,30,34}$ OIT was administered as daily oral doses of peanut flour mixed with yoghurt or in a food vehicle chosen by the subjects; in the remaining three studies ${ }^{31-33}$ it was not clear how peanut was administered. Three studies ${ }^{29,32,34}$ started the treatment with an open peanut challenge to determine the threshold dose of peanut exposure, two studies ${ }^{30,31}$ started with a DBPCFC, and the remaining study did not report on any challenge at the outset of the study. ${ }^{33}$

\section{Study quality assessment}

All the included studies were case series so the risk of bias was high. None of the studies reported consecutive recruitment of participants, so the risk of selection bias was particularly high. Because there was no blinding of patients or clinicians/researchers, the risk of information bias was also high. There is also an inherently high risk of publication bias with case series.

\section{Effectiveness and safety of OIT}

\section{Threshold dose of peanut exposure}

In interpreting these data it is important to appreciate that each whole peanut contains approximately 200-250mg peanut protein and that $2 \mathrm{mg}$ peanut flour $=1 \mathrm{mg}$ peanut protein.

Four studies ${ }^{29,30,31,34}$ assessed changes in the threshold dose of peanut required to provoke symptoms as an outcome. One study ${ }^{32}$ used the ability to tolerate one peanut as an outcome and the remaining study used the ability to tolerate $7.8 \mathrm{~g}$ of peanut flour as an outcome..$^{33}$

Three of these studies ${ }^{29-31}$ compared the threshold dose for peanut allergen at the beginning of the study with the threshold dose during the maintenance phase. In the study by Jones et al. ${ }^{29}$ symptoms occurred on the initial day in 39 participants at a median dose of $6 \mathrm{mg}$ peanut protein (range $0.1-50 \mathrm{mg}$ ) whilst, at the final OFC, symptoms started in 29 of these participants at a median dose of $1.8 \mathrm{~g}$ peanut protein, a mean increase of 300 times the initial tolerated dose. Twenty-seven of the 29 participants who completed the study reached the total peanut target dose of $3.9 \mathrm{~g}$ (equating to approximately 16 peanuts).

In the study by Clark et al. ${ }^{30}$ four participants with peanut allergy underwent OIT. At the initial assessment one participant had a threshold dose of $5 \mathrm{mg}$ peanut protein (approximately $1 / 50$ of a peanut) whilst the other three participants each had a threshold dose of $50 \mathrm{mg}$ (approximately $1 / 5$ of a peanut). At the post-intervention challenge the highest tolerated dose ranged from $2.39 \mathrm{~g}$ to $2.76 \mathrm{~g}$ (i.e. $10-12$ peanuts), an increase of between 48 and 478 times the initial threshold dose.

Blumchen et al. ${ }^{31}$ initially performed a rush OIT protocol with whole crushed roasted peanuts (which was presented in boiled apple). Patients who tolerated 500mg peanut or more after the rush protocol went directly to the maintenance phase for 8 weeks. If a dose of 500mg whole peanut was not achieved during this initial rush phase, patients were discharged from hospital and were transferred onto the longerterm build-up protocol (0-20 months) which was delivered in an outpatient setting. In 17 of 22 patients the amount of peanut tolerated did not change during the rush OIT compared with the initial food challenge. These patients were discharged and post-rush OIT doses were taken on a daily basis (range 24$400 \mathrm{mg}$ peanut) until they reached the goal of at least $500 \mathrm{mg}$ peanut. Five patients reached a dose of 500mg peanut (median $1 \mathrm{~g}$, range $0.9-3 \mathrm{~g}$ ) and went to the maintenance phase for 8 weeks. Fourteen of 23 patients $(61 \%)$ reached the protective dose of $500 \mathrm{mg}$ whole peanut. At the final DBPCFC, patients tolerated a median of $1 \mathrm{~g}$ peanut (range $0.25-4 \mathrm{~g}$ ) in 
comparison with $0.19 \mathrm{~g}$ peanut at the DBPCFC before the start of OIT (range $0.02-1 \mathrm{~g}$ ).

Two studies reported only on the final dose of peanut protein that patients were able to consume without provoking symptoms: Nash et al..$^{33}$ reported that all 13 participants who completed the study were able to consume a final dose of $7.8 \mathrm{~g}$ peanut flour (approximately 31 peanuts) safely, although five of the 13 patients experienced mild symptoms (discussed below). Wasserman et al..$^{32}$ reported that 16 participants were able to tolerate at least one peanut; these data are, however, difficult to interpret because the number of patients with peanut allergy who received OIT is unclear from the report $(n=16-38)$. Finally, Buchanan et al. ${ }^{34}$ did not report any threshold doses or changes in the threshold dose of peanut during OIT.

None of the studies provided any data on whether tolerance - i.e. the ability to consume peanuts safely when off treatment - had been achieved.

\section{Adverse events}

All six papers ${ }^{29-34}$ reported adverse events during administration of the OIT protocol. Table 3 provides a summary of the severity of adverse events in each of these studies. ${ }^{28}$ Most of the adverse events that occurred during these studies were treated with medication (e.g. antihistamines and adrenaline). The use of medication during OIT was evaluated in five studies. ${ }^{29,30,32-34}$ One study ${ }^{31}$ did not report the use of medication.

In the study by Blumchen et al., ${ }^{31}$ allergic reactions occurred frequently during the rush OIT protocol. Grade 1-3 reactions were reported with $4 / 6(67 \%)$ of the participants developing hives, 3/6 (50\%) experiencing vomiting, 1/6 (17\%) developing diarrhoea, and 3/6 (50\%) experiencing coughing.

In the study by Buchanan et al. ${ }^{34}$ during the initial rush phase $57 \%$ of the participants required a single dose of antihistamine to treat symptoms. None of the subjects required adrenaline. No detailed information about medication use during the maintenance phase was given

Clark et al. ${ }^{30}$ reported grade $1-4$ reactions during their OIT protocol. During the initial challenge one participant developed anaphylaxis with sudden onset of severe abdominal pain, wheezing, breathlessness, and a fall in peak expiratory flow; this reaction was treated with intramuscular adrenaline, nebulised salbutamol, intravenous chlorphenamine, and intravenous hydrocortisone. The remaining three patients also developed reactions during these pre-intervention challenges that required treatment with antihistamines alone. Three of the four patients (75\%) subsequently experienced some adverse events during the build-up phase, although the majority were mild: two (50\%) experienced abdominal pain, two (50\%) developed oral itching, two (50\%) developed erythema, one developed nausea and vomiting, and three (75\%) developed rhino-conjunctivitis.

In the study by Jones et al., ${ }^{29} 36 / 39$ participants (92\%) experienced some symptoms during the initial escalation day. Upper respiratory symptoms were most common with 27/39 participants (69\%) complaining of mild sneezing/itching and
Table 3. Severity of adverse reactions

\begin{tabular}{|c|c|c|c|c|}
\hline $\begin{array}{l}\text { Study, } \\
\text { reference }\end{array}$ & Grade 1 & Grade 2 & Grade 3 & Grade 4 \\
\hline $\begin{array}{l}\text { Blumchen } \\
\text { et } a l . .^{31}\end{array}$ & + & + & + & - \\
\hline $\begin{array}{l}\text { Buchanan } \\
\text { et al. }{ }^{34}\end{array}$ & $\begin{array}{l}\text { Not } \\
\text { detailed }\end{array}$ & $\begin{array}{l}\text { Not } \\
\text { detailed }\end{array}$ & $\begin{array}{l}\text { Not } \\
\text { detailed }\end{array}$ & $\begin{array}{l}\text { Not } \\
\text { detailed }\end{array}$ \\
\hline Clark et al..$^{30}$ & + & + & + & + \\
\hline Jones et al. ${ }^{29}$ & + & + & + & - \\
\hline Nash et al..$^{33}$ & + & + & + & - \\
\hline $\begin{array}{l}\text { Wasserman } \\
\text { et al. }{ }^{32}\end{array}$ & $\begin{array}{l}\text { Not } \\
\text { detailed }\end{array}$ & $\begin{array}{l}\text { Not } \\
\text { detailed }\end{array}$ & $\begin{array}{l}\text { Not } \\
\text { detailed }\end{array}$ & $\begin{array}{l}\text { Not } \\
\text { detailed }\end{array}$ \\
\hline
\end{tabular}

laryngeal symptoms. Furthermore, 17/39 participants (44\%) developed nausea and abdominal pain, 8/39 (21\%) developed mild diarrhoea or vomiting, 24/39 (62\%) developed mild-tomoderate cutaneous symptoms, and 6/39 (15\%) experienced respiratory symptoms. During the build-up phase, patients experienced symptoms after $46 \%$ of build-up doses. All participants experienced some minor symptoms at one or more points in the home dosing schedule $(3.7 \%$ of 14,773 doses given), of which upper respiratory (1.2\%) and skin symptoms $(1.1 \%)$ were the most common. During the final open OFC, $27 / 29(93 \%)$ of completing patients reached the total peanut dose of $3.9 \mathrm{~g}$ with no more than mild symptoms. One participant did not reach the final dose because of mild urticaria and an episode of vomiting. The authors mentioned that treatment was given in $0.8 \%$ of home doses during the build-up/maintenance phase. Two participants received adrenaline. No further specification of medication use during this or other treatment phases was given.

In the study by Nash et al. ${ }^{33}$ most subjects had mild allergic symptoms during the initial quick build-up phase. However, two patients experienced significant systemic allergic symptoms during this phase; no information was given about whether treatment with adrenaline was needed. Five of the 13 participants (38\%) who completed the study experienced mild symptoms during the final OFC, with four (31\%) needing treatment with antihistamines.

Wasserman et al..$^{32}$ reported that significant reactions occurred in at least seven peanut-treated patients (denominator unclear); these reactions required treatment with a single dose of adrenaline.

\section{Drop-outs}

Four studies reported on the number of drop-outs. ${ }^{29-32}$ There were no drop-outs in the studies by Clark et al. ${ }^{30}$ or Blumchen et al. ${ }^{31}$ Both studies had a small number of participants $(n=4$ and $\mathrm{n}=6$, respectively). During the study by Jones et al., ${ }^{29} 10$ patients (25\%) dropped out after the initial day, of whom six discontinued treatment for personal reasons (e.g. transport issues and parental anxiety). The allergic reactions experienced by these participants during the initial day were comparable to the reactions of other participants. The four other participants 
who discontinued did so because of allergic reactions to OIT that did not resolve with continued treatment or dose reduction; three had gastrointestinal complaints and one participant had symptoms of asthma. In the study by Wasserman et al..$^{32}$ nine participants $(18 \%)$ of the total study population dropped out, but it is not clear how many of these occurred in patients allergic to peanut (as opposed to those being treated for other food allergies). In the remaining two studies it was unclear if there were any drop-outs. ${ }^{33,34}$

Other outcomes

None of the studies provided data on any of the other outcomes of interest (i.e. patient/carer satisfaction, impact on quality of life, healthcare utilisation, or cost-effectiveness). Data on immunological outcomes are shown in Appendix 7 (see Supplementary materials available at www.thepcrj.org).

\section{Discussion}

After a wide-ranging search of the published and unpublished literature, we found six case series that satisfied our inclusion criteria. This evidence shows that OIT has the potential to raise the threshold for peanut allergen exposure, but these data do need to be interpreted with caution as they come from studies employing weak designs that render them at high risk of bias. In the studies by Jones et al., ${ }^{29}$ Clark et al., ${ }^{30}$ and Nash et al., ${ }^{33}$ the peanut threshold dose increased several fold. The threshold dose reached in these studies is substantially higher than that likely to be encountered during accidental ingestion. This suggests that OIT may be effective in inducing desensitisation. All three of these studies used a three-phase OIT protocol. In contrast, the rush protocol of Blumchen et al. ${ }^{31}$ was not effective in raising the threshold dose. It could be argued that the lack of evidence of benefit in the study by Blumchen et al. may have been because all the participants in this study had asthma and were thus at particularly high risk of anaphylactic reactions. On the other hand, all the participants in the study by Clark et al. ${ }^{30}$ were also at high risk of anaphylaxis but the threshold dose of the participants in this study was effectively raised by OIT. The study by Wasserman et al. ${ }^{32}$ was the only one performed in an officebased setting. This study also differed from the other studies in other respects; for example, OIT was given during a two-phase protocol and increasing doses were given every 4 days. This is in contrast to some of the other studies ${ }^{29,30,34}$ where doses were increased every 2 weeks. Wasserman et al. ${ }^{32}$ reported that, because of their OIT protocol, 16 participants could tolerate $\geq 1$ peanut, but it may be questioned if this threshold dose is enough for protection during accidental peanut ingestion. Based on the included studies, it appears that a three-phase therapy with slow increasing doses of peanut allergen is the most promising approach in raising the threshold dose for peanut allergen.

Of concern was the finding that adverse events associated with OIT were observed in each of the studies. Grade 1-3 reactions occurred in three studies. ${ }^{29,31,33}$ Symptoms were experienced particularly frequently during the rush protocol in the study by Blumchen et al. ${ }^{31}$ and in the other studies ${ }^{29,33}$ during the initial escalation phase. Such reactions appeared to be most frequent during rapid up-dosing, emphasising the importance of taking due care when undertaking this phase of the OIT protocol.

Adrenaline was used in three studies. ${ }^{29,30,32}$ In the study by Clark et al., ${ }^{30}$ one participant had an anaphylactic reaction during the initial escalation day; this participant fully recovered after adequate treatment and continued with immunotherapy. In the study by Jones et al., ${ }^{29}$ two participants received adrenaline once after home dosing. It was unclear how many times anaphylaxis occurred in the study by Wasserman et al., ${ }^{32}$ but an appreciable number of these patients experienced reactions that were treated with adrenaline.

\section{Limitations of this review}

This review has been conducted relatively shortly after renewed interest in the field of OIT for food allergy and, as such, it is based on a limited number of methodologically weak studies which are potentially at high risk of bias and confounding, some of which have as yet only reported at scientific meetings. The rationale for undertaking early systematic reviews is now well recognised in that such reviews have, among other things, the potential to influence the commissioning and design of future primary studies. M oreover, we hope that, as the first systematic review of OIT for the management of food allergy, this work will also serve as a foundation for and inform future systematic reviews in relation to other important food groups (e.g. milk, eggs, tree nuts, etc). Any conclusions drawn must therefore be seen as preliminary at this stage. Additional limitations include the fact that these studies are of relatively short duration so it is not possible to generate any evidence on whether or not OIT can result in a state of tolerance. It should also be borne in mind that case series are particularly prone to publication bias; it is therefore possible that there are similar unpublished case series that we were not able to find despite our attempts to locate unpublished material. If so, this would mean that our findings suggest an over-optimistic assessment of the possible benefits associated with OIT. In keeping with the approach we have used in other disease contexts, ${ }^{35-37}$ we plan to update this review at regular intervals which will allow us to incorporate studies that have been published after the cut-off date for our searches and thereby maintain oversight of key developments in this important field of enquiry.

\section{Conclusions}

Our systematic review suggests that, in a subset of patients with peanut allergy, OIT may prove effective in raising the threshold of exposure to substantially higher doses than those that tend to be encountered through accidental exposure. Rush OIT-based approaches appear less efficacious than the more gradual threephase treatment approach. Although side-effects clearly occur, OIT appears to be relatively safe if administered in carefully monitored clinical settings with gradually escalating doses. It seems that the majority of these adverse events can be effectively treated with antihistamines, although adrenaline should be 
available at all times.

Overall, OIT appears to be a promising new therapeutic approach, but its effectiveness, cost-effectiveness, and risk profile now need to be better established. We therefore recommend that OIT administration should, for the present, only take place in clinical trial settings.

\section{Handling editor Paul Stephenson}

Conflicts of interest AS is a consultant to ALK and Phadia. He has advised the Scottish Government and House of Lords Allergy Inquiry. He is the Royal College of General Practitioners Clinical Champion in Allergy. AS is the Joint Editor-in-Chief of the PCRJ, but was not involved in the editorial review of, nor the decision to publish, this article.

Contributorship AS conceived this study. IV and UN undertook searches, and together with AS and EB critically appraised studies. IV, UN and EB undertook data extraction, with IV, UN, EB and AS leading the analysis. IV and AS drafted the manuscript and all authors commented on drafts of the article. AS was the PI for this project.

Funding Chief Scientist's Office of the Scottish Government.

\section{References}

1. Al-Muhsen $\mathrm{S}$, Clarke AE, Kagan RS. Peanut allergy: an overview. CMAJ 2003;168:1279-85

2. Kotz $D$, Simpson $C R$, Sheikh A. Incidence, prevalence, and trends of general practitioner-recorded diagnosis of peanut allergy in England, 2001 to 2005. J Allergy Clin Immunol 2011 Jan 12 [Epub ahead of print].

3. Worth A, Soar J, Sheikh A. Management of anaphylaxis in the emergency setting. Expert Rev Clin Immunol 2010;6:89-100. http://dx.doi.org/10.1586/eci.09.73

4. Sicherer SH. Clinical update on peanut allergy. Ann Allergy Asthma Immunol 2002;88:350-61. http://dx.doi.org/10.1016/S1081-1206(10)62363-0

5. Frank L, Marian A, Visser M, Weinberg E, Potter PC. Exposure to peanuts in utero and in infancy and the development of sensitization to peanut allergens in young children. Pediatr Allergy Immunol 1999;10:27-32. http://dx.doi.org/10.1034/.1399-3038.1999.101010.x

6. McLean $S$, Sheikh A. Does avoidance of peanuts in early life reduce the risk of peanut allergy? BMJ 2010;340:c424. http://dx.doi.org/10.1136/bmj.c424

7. Lack G, Fox D, Northstone K, Golding J. Avon Longitudinal Study of Parents and Children Study Team. Factors associated with the development of peanut allergy in childhood. N Engl J Med 2003;348:977-85. http://dx.doi.org/10.1056/ NEJM oa013536

8. Worth A, Sheikh A. Food allergy and atopic eczema. Curr Opin Allergy Clin Immunol 2010;10:226-30. http://dx.doi.org/10.1097/ACl.0b013e3283387fae

9. van den Oord RA, Sheikh A. Filaggrin gene defects and risk of developing allergic sensitisation and allergic disorders: systematic review and meta-analysis. BMJ 2009;339:b2433. http://dx.doi.org/10.1136/bmj.b2433

10. Pansare M, Kamat D. Peanut allergies in children: a review. Clin Pediatr (Phila) 2009;48:709-14. http://dx.doi.org/10.1177/0009922808330782

11. Sheikh A, Walker S. 10-minute consultation. Food allergy. BMJ 2002;325:1337. http://dx.doi.org/10.1136/bmj.325.7376.1337

12. Sicherer SH, Burks AW, Sampson HA. Clinical features of acute allergic reactions to peanut and tree nuts in children. Pediatrics 1998;102:e6. http://dx.doi.org/10.1542/peds.102.1.e6

13. Schäppi GF, Konrad V, Imhof D, Etter R, Wüthrich B. Hidden peanut allergens detected in various foods: findings and legal measures. Allergy 2001;56:1216-20. http://dx.doi.org/10.1034/j.1398-9995.2001.00280.x

14. Hourihane JO'B, Kilburn SA, Nordlee JA, Hefle SL, Taylor SL, Warner JO. An evaluation of the sensitivity of subjects with peanut allergy to very low doses of peanut protein: a randomized, double-blind, placebo-controlled food challenge study. J Allergy Clin Immunol 1997;100:596-600.

http://dx.doi.org/10.1016/S0091-6749(97)70161-1
15. Wensing M, Penninks AH, Hefle SL, Koppelman SJ, Bruijnzeel-Koomen CA, Knulst AC. The distribution of individual threshold doses eliciting allergic reactions in a population with peanut allergy. J Allergy Clin Immunol 2002;110:915-20. http://dx.doi.org/10.1067/mai.2002.129235

16. Akeson N, Worth A, Sheikh A. The psychosocial impact of anaphylaxis on young people and their parents. Clin Exp Allergy 2007;37:1213-20. http://dx.doi.org/10.1111/j.1365-2222.2007.02758.x

17. Soar J, Pumphrey R, Cant A, et al. Emergency treatment of anaphylactic reactions - guidelines for healthcare providers. Resuscitation 2008;77:157-69. http://dx.doi.org/10.1016/j.resuscitation.2008.02.001

18. Biarent D, Bingham R, Richmond $S$, et al. European Resuscitation Council Guidelines for Resuscitation 2005: Section 6. Paediatric life support. Resuscitation 2005;67S1:S97-S133.

19. Schofield AT. A case of egg poisoning. Lancet 1908;1:716. http://dx.doi.org/10.1016/S0140-6736(00)67313-0

20. Calderon MA, Alves B, Jacobson M, Hurwitz B, Sheikh A, Durham A. Allergen injection immunotherapy for seasonal allergic rhinitis. Cochrane Database Syst Rev 2007;1:CD001936. http://dx.doi.org/10.1002/14651858.CD001936.pub2.

21. Bilo MB, Severino M, Cilia M, et al. The VISYT trial: venom immunotherapy safety and tolerability with purified vs nonpurified extracts. Ann Allergy Asthma Immuno 2009;103:57-61. http://dx.doi.org/10.1016/S1081-1206(10)60144-5

22. Nelson HS, Lahr J, Rule R, Bock A, Leung D. Treatment of anaphylactic sensitivity to peanuts by immunotherapy with injections of aqueous peanut extract. J Allergy Clin Immunol 1997;99(Suppl):744-51. http://dx.doi.org/10.1016/S0091-6749(97)80006-1

23. Bird JA, Burks AW. Food allergy and asthma. Prim Care Respir J 2009;18:258-65 http://dx.doi.org/10.4104/pcrj.2009.00036

24. Higgins JPT, Green S. Cochrane Handbook for Systematic Reviews of Interventions. Version 5.0.2 (Chapter 11, Section 11.2) [updated September 2009].

25. Cochrane Collaboration. Non-randomised controlled study (NRS) designs. Available at: http://www.ccg.cochrane.org/non-randomised-controlled-study-nrs-designs.

26. Heinzerling L, Frew AJ, Bindslev-Jensen C, et al. Standard skin prick testing and sensitisation to inhalant allergens across Europe - a survey from GALEN network. Allergy 2006;60:1287-300. http://dx.doi.org/10.1111/j.1398-9995.2005.00895.x

27. Clark AT, Ewan PW. Interpretation of tests for nut allergy in one thousand patients, in relation to allergy or tolerance. Clin Exp Allergy 2003;33:1041-5. http://dx.doi.org/10.1046/j.1365-2745.2003.01624.x

28. Malling HJ, Weeke B. Position paper: Immunotherapy. Allergy 1993;48:9-35. http://dx.doi.org/10.1111/j.1398-9995.1993.tb04754.x

29. Jones $S M$, Pons $L$, Roberts $J$, et al. Clinical efficacy and immune regulation with peanut oral immunotherapy. J Allergy Clin Immunol 2009;124:292-300. http://dx.doi.org/10.1016/j.jaci.2009.05.022

30. Clark AT, Islam S, King Y, Deighton J, Anagnostou K, Ewan PW. Successful oral tolerance induction in severe peanut allergy. Allergy 2009;64:1218-20.

31. Blumchen $\mathrm{K}, \mathrm{Ulbricht} \mathrm{H}$, Staden $\mathrm{U}$, et al. Oral peanut immunotherapy in children with peanut anaphylaxis. J Allergy Clin Immunol 2010;126:83-91. http://dx.doi.org/10.1016/j.jaci.2010.04.030

32. Wasserman RL, Mansfield LE, Gallucci AR, et al. Office based oral desensitization of patients with anaphylactic sensitivity to foods is safe and effective. J Allergy Clin Immunol 2010;125:AB59(233).

33. Nash SD, Steele PH, Kamilaris JS, et al. Oral immunotherapy for children with peanut allergy. J Allergy Clin Immunol 2008;121:S136. http://dx.doi.org/10.1016/j.jaci.2007.12.544

34. Buchanan A, Scurlock AM, Jones SM, et al. Oral desensitization and induction of tolerance in peanut-allergic children. J Allergy Clin Immunol 2006;117:S327. http://dx.doi.org/10.1016/j.jaci.2005.12.1290

35. Sheikh A, Hurwitz B. House dust mite avoidance measures for perennial allergic rhinitis. Cochrane Database Syst Rev 2001;4:CD001563.

36. Sheikh A, Hurwitz B, Shehata Y. House dust mite avoidance measures for perennial allergic rhinitis. Cochrane Database Syst Rev 2007;1:CD001563.

37. Sheikh A, Hurwitz B, Nurmatov U, van Schayck CP. House dust mite avoidance measures for perennial allergic rhinitis (update). Cochrane Database Syst Rev 2010;7:CD001563. 
Appendix 1: Working definitions for eligible study designs

- Randomised controlled trials: all participants are allocated at random (e.g. random number sequence) to receive a treatment or a control intervention, whether placebo or active.

- Quasi-randomised controlled trials: the intervention is allocated in a way that is not truly random (e.g. allocation by birth, day of the week, month of the year).

- Controlled clinical trials: trials that contain (at least) two groups, where one receives the treatment and the other group is a comparison group. The comparison group receives a placebo, another treatment, or no treatment at all.

- Controlled before-and-after design: a study in which observations are made before and after the implementation of an intervention, both in a group that receives the intervention and in a control group that does not.

- Interrupted time series: a study that uses observations at multiple time points before and after an intervention. The design attempts to detect whether the intervention has had an effect significantly greater than any underlying trend over time. There are two minimum criteria for inclusion of interrupted time series designs: (i) a clearly defined point in time when the intervention occurred; (ii) at least three data points before and three after the intervention.

- Case series: observations are made on a series of individuals, usually all receiving the same intervention, before and after an intervention but with no control group. Only case series with a minimum of three cases were eligible for inclusion. 
Appendix 2: Search strategy for CENTRAL and The Cochrane Library

\#1 MeSH descriptor Anaphylaxis explode all trees

\#2 anaph?|* or (acute near allerg*)

\#3 (\#1 OR \#2)

\#4 MeSH descriptor Hydrocortisone explode all trees

\#5 MeSH descriptor Prednisolone explode all trees

\#6 MeSH descriptor Betamethasone explode all trees

\#7 MeSH descriptor Clobetasol explode all trees

\#8 MeSH descriptor Cortisone explode all trees

\#9 MeSH descriptor Anti-Inflammatory Agents explode all trees

\#10 MeSH descriptor Immunosuppressive Agents explode all trees

\#11 MeSH descriptor Pregnenediones explode all trees

\#12 MeSH descriptor Glucocorticoids explode all trees

\#13 MeSH descriptor Triamcinolone Acetonide explode all trees

\#14 MeSH descriptor Triamcinolone explode all trees

\#15 MeSH descriptor Drug Hypersensitivity explode all trees

\#16 prednisolone or betamethasone or cortison* or deflazacort or calcort or dexamethasone or hydrocortisone or efcortesol or hydrocortone or solu-cortef or methylprednisolone or solu-medrone or depo-medrone or riamcinolone or kenalog

\#17 (\#4 OR \#5 OR \#6 OR \#7 OR \#8 OR \#9 OR \#10 OR \#11 OR \#12 OR \#13 OR \#14 OR \#15 OR \#16)

\#18 (\#3 AND \#17) 
Appendix 3: Search Strategy for MEDLINE (Ovid SP)

\#1. exp Anaphylaxis/

\#2. (anaph?!* or (acute adj3 allerg*)).mp.

\#3. \#1 or \#2

\#4. exp Hydrocortisone/ or exp Prednisolone/ or exp Betamethasone 17-Valerate/ or exp Betamethasone/ or exp Clobetasol/ or exp Cortisone/ or exp Anti-Inflammatory Agents/ or exp Immunosuppressive Agents/ or exp Pregnenediones/ or exp Glucocorticoids/ or exp Prednisone/ or exp Dexamethasone Isonicotinate/ or exp Dexamethasone/ or exp Steroids/ or exp Methylprednisolone/ or exp Triamcinolone Acetonide/ or exp Triamcinolone/ or exp Drug Hypersensitivity/

\#5. (prednisolone or betamethasone or cortison* or deflazacort or calcort or dexamethasone or hydrocortisone or efcortesol or hydrocortone or solu-cortef or methylprednisolone or solu-medrone or depo-medrone or triamcinolone or kenalog).mp.

\#6. \#4 or \#5

\#7. \#3 and \#6

\#8. ((randomized controlled trial or controlled clinical trial).pt. or randomized.ab. or placebo.ab. or clinical trials as topic.sh. or randomly.ab. or trial.ti.) not (animals not (humans and animals)).sh.

\#9. \#7 and \#8 
Appendix 4: Search strategy for EMBASE (Ovid SP)

\#1. exp anaphylaxis/

\#2. (anaph?|* or (acute adj3 allerg*)).mp.

\#3. \#1 or \#2

\#4. hydrocortisone/ or prednisolone/ or betamethasone valerate/ or betamethasone/ or clobetasol/ or cortisone/ or antiinflammatory agent/ or pregnane derivative/ or glucocorticoid/ or prednisone/ or dexamethasone isonicotinate/ or dexamethasone/ or steroid/ or methylprednisolone/ or triamcinolone acetonide/ or triamcinolone/ or drug hypersensitivity/

\#5. (prednisolone or betamethasone or cortison* or deflazacort or calcort or dexamethasone or hydrocortisone or efcortesol or hydrocortone or solu-cortef or methylprednisolone or solu-medrone or depo-medrone or triamcinolone or kenalog).ti,ab.

\#6. \#4 or \#5

\#7. \#3 and \#6

\#8. (placebo.sh. or controlled study.ab. or random*.ti,ab. or trial*.ti,ab.) not (animals not (humans and animals)).sh.

\#9. \#7 and \#8 
Appendix 5: Search strategy for CINAHL (EBSCOhost)

S1 (MM "Anaphylaxis")

S2 TX anaphyl* or (acute allerg*)

S3 S1 or S2

S4 TX (prednisolone or betamethasone or cortison* or deflazacort or calcort or dexamethasone or hydrocortisone or efcortesol or hydrocortone or solu-cortef or methylprednisolone or solu-medrone or depo-medrone or triamcinolone or kenalog)

S5 (MM "Hydrocortisone") or (MM "Prednisolone") or (MM "Betamethasone") or (MM "Cortisone") or (MM "Antiinflammatory Agents") or (MH "Immunosuppressive Agents+") or (MH "Glucocorticoids") or (MM "Dexamethasone") or (MM "Steroids") or (MM "Methylprednisolone") or (MM "Triamcinolone") or (MH "Drug Hypersensitivity+")

S6 S4 or S5

S7 S3 and S6

S8 TX random* or trial* or multicenter* or ((double or single or triple) and (mask* or blind*)) or placebo* S9 S7 and S8 
Appendix 6: Experts contacted

\begin{tabular}{|lll|}
\hline Name of author & Country & Email address \\
\hline Dr Kirsten Beyer & Germany & Kirsten.Beyer@charite.de \\
\hline Prof Wesley Burks & USA & Wesley.Burks@duke.edu \\
\hline Dr Andrew T Clark & UK & AtClark@doctors.org.uk \\
\hline
\end{tabular}


Appendix 7: Immunological changes

Four studies $29,32-34$ evaluated the changes in peanut-specific lgE; two of these studies ${ }^{29,34}$ also investigated changes in peanut-specific $\operatorname{lgG}$. One of these also evaluated changes in $\lg G 4$ and evaluated other immunological changes (i.e. basophils and regulatory $T$ cells); this study also appraised the change in sensitisation as judged by skin prick test results during OIT ${ }^{29}$

Jones et al. ${ }^{29}$ found a median peanut-specific IgE on the initial study day of 85kU/L (range 9.1-840.0). The median peanut-specific IgE had increased to $249.0 \mathrm{kU} / \mathrm{L}$ after 3 months. The median peanut-specific IgE then decreased at 12 and 18 months, so there was no significant difference in specific IgE from baseline. Peanut-specific lgE then significantly decreased until 33 months of treatment $(p<0.0005)$. Nash et al..$^{33}$ also reported similar changes in specific IgE, observing that the initial mean specific IgE was $184 \mathrm{kU} / \mathrm{L}$. Peanut-specific IgE levels then increased significantly at 3 months $(p=0.016)$ and 6 months ( $p=0.039$ ) before declining. In contrast, Buchanan et al. ${ }^{34}$ found that at 6 months the mean peanutspecific lgE was unchanged $(p=0.6)$ from the initial mean of $140 \mathrm{kU} / \mathrm{L}$. Wasserman et al. ${ }^{32}$ reported only the mean peanut-specific IgE at the end of treatment $(57 \mathrm{kU} / \mathrm{L})$; it should be noted, however, that this mean specific lgE level was derived only from those participants $(n=16)$ who were able to tolerate $\geq 1$ peanut at the end of the study. Clark et al. ${ }^{30}$ reported the peanut-specific IgE levels at the beginning of the study; for two participants these levels were $>100 \mathrm{kU} / \mathrm{L}$ and for the other two participants they were $6 \mathrm{kU} / \mathrm{L}$ and $16 \mathrm{kU} / \mathrm{L}$, respectively.

Most of the studies also evaluated other immunological changes. Three studies ${ }^{29,33,34}$ evaluated the changes in peanut-specific lgG during OIT. Jones et al. ${ }^{29}$ found a median baseline serum peanut-specific $\operatorname{lgG}$ of $9.7 \mathrm{mg} / \mathrm{L}$ (range $2.5-56.0$ ). A significant increase $(p<0.0005)$ in specific lgG levels was seen by 3 months; IgG levels remained increased from baseline until 24 months and then, whilst still receiving treatment, this gradually returned to the baseline level over the next 11 months. Nash et al. ${ }^{33}$ found the same initial increase; peanut-specific lgG increased significantly from baseline at 3 months $(p=0.016)$ and at 8 months ( $p=0.033$ ). These results were supported by Buchanan et al. ${ }^{34}$ At baseline they found a mean peanut-specific $\lg$ of $14 \mathrm{kU} / \mathrm{L}$; at 6 months $\lg G$ was increased over twofold ( $p=0.0002$ ).

Jones et al. ${ }^{29}$ found an initial median peanut-specific lgG4 of $0.3 \mathrm{mg} / \mathrm{L}$ (range $0.1-0.4$ ). After 3 months of OIT, IgG4 concentrations were significantly increased to a median concentration of $2.0 \mathrm{mg} / \mathrm{L}(\mathrm{p}<0.005)$ and these then remained elevated until the end of the study $(p<0.005)$. This research group also measured changes in a range of cytokines and found that interleukins IL-1 $13, \mathrm{LL}-5, \mathrm{IL}-10$, tumour necrosis factor $\alpha$, macrophage inflammatory protein 1 , and growth factors G-CSF and GM-CSF all significantly increased over 24 months of treatment. Changes in FoxP3 ${ }^{+}$T cells were investigated in a subset $(n=10)$ of patients who received OIT for 36 months and this analysis found a 1.5-fold $(p<0.05)$ increase in the number of FoxP3+ $T$ cells at both 6 and 12 months, but with a subsequent decrease to baseline by 20 months. 